transcribed and analysed for IBD outcomes elicited by clinicians (or volunteered by patients), including specific items required for Harvey-Bradshaw Index (HBI) and Simple Clinical Colitis Activity Index (SCCAI).

Results Most commonly elicited outcomes are shown in the table 1. HBI or SCCAI were collected in only $4(8 \%)$ of consultations. In the remainder, domains of HBI and SCCAI were discussed in variable detail. Complete HBI coverage: 5/29 (17\%); symptom components of HBI (wellbeing, liquid stools, abdominal pain): 16/29 (55\%). No Crohn's disease consultation involved specific discussions about symptoms over past 24 hrs (including 2 where HBI was calculated). Complete SCCAI coverage: only 1 consultation. Partial coverage (5 out of 6 SCCAI domains): 8/21 (38\%). Symptoms were never specifically defined over past 3 days. Certain symptoms were elicited significantly more often by nurses than doctors $(p<0.05)$, and coverage varied by disease severity. Interviews are under way to explore views, barriers and facilitators to standardisation of outcomes assessment.

Conclusions There is high variability in breadth, depth and quantification of outcomes during routine clinical assessments. Most domains for activity indices were elicited but formal scoring and assessment for a fixed time period was rare. Standardised outcomes may be better-captured directly from patients (PROMs) than via clinician-generated indices.

\section{PTH-116 VEDOLIZUMAB IN THE TREATMENT OF CROHN'S DISEASE AND ULCERATIVE COLITIS - NON-TERTIARY REAL WORLD ANALYSIS}

Susan Ritchie*, Frances Maw, Mohammed Hussien, Victoria Fletcher, Linda Crissop, Sarah Jordan, Anjan Dhar. County Durham and Darlington NHS Foundation Trust And Durham University, Darlington, UK

\subsection{6/gutjnl-2019-BSGAbstracts. 175}

Introduction Vedolizumab is licenced for treatment of moderate-to-severe ulcerative colitis(UC) and Crohn's disease(CD) after failure of conventional or anti-TNF therapy. Clinical trial data from the GEMINI studies reported a Wk 6 response rate of $47 \%$ in UC and $31 \%$ in $\mathrm{CD}$ and $\mathrm{Wk} 52$ response rate of $42-57 \%$ (UC) and 39-44\% (CD). Data from expert centres report a lower 12-month remission of $26-33 \%$, with endoscopic mucosal healing of upto $63 \%$. Prior anti-TNF exposure pts are likely to have a worse outcome.(VICTORY Study)

Aims This single non tertiary study was designed to compare clinical, biochemical and endoscopic outcomes of Vedolizumab treatment in UC and CD. A secondary aim was to compare outcomes for anti-TNF naïve v. anti-TNF exposed pts.

Methods All IBD pts eligible for Vedolizumab at MDT review between 2016-2018 were included. Pts were grouped into anti-TNF naïve and anti-TNF exposed, the severity of UC and CD classified by Simple colitis index or Harvey Bradshaw Index respectively, extent of disease defined by Montreal Classification. Baseline demographics, serum C-reactive protein, faecal calprotectin, colonoscopic severity (Mayo Endoscopic score and SES-CD) were recorded before starting Vedolizumab treatment and at 12 months. Standard definitions were used for clinical and endoscopic remission.

Results 35 patients (20 UC,15 CD), M:F 21:14(UC13:7, CD8:7) were included. The mean age was $46.15 \mathrm{y}$ for UC (22-77) and 49.4y for CD (26-79). 15/20 UC patients and $12 / 15$ CD patients were anti-TNF exposed. In the UC group: disease extent was $\mathrm{E} 1=1, \mathrm{E} 2=5$, and $\mathrm{E} 3=14$; mean SCAI 3.15, mean $\mathrm{CRP}=23 \mathrm{mg} / \mathrm{L}$ (range4-202), mean faecal calprotectin 616ug/g (range 4-1684), endoscopic severity mild $=4$, moderate $=10$, severe $=6$. At 12 months, 9/15(60\%) anti-TNF exposed and $2 / 5$ anti TNF naive (40\%) were in clinical remission, with endoscopic remission in 4 and 1 pt respectively. Mean CRP was 5 (4-7) and mean FCP 472. 1 pt developed a rash, 1 pt had abnormal LFT, and 2 failed to respond requiring surgery. In the $\mathrm{CD}$ group: mean Harvey Bradshaw score was 15 (0-30), mean CRP 15.7(4-202), FCP 250 (5683). Disease extent was $\mathrm{L} 1=2, \mathrm{~L} 2=4$ and $\mathrm{L} 3=9$. At 12 months 6/12 (50\%) anti TNF exposed patients and 2/3(66\%) anti TNF naïve pts were in clinical remission with mean CRP 7.8(4-15) and FCP 472(4-1957).

Conclusion In this real world study, we found that both UC and CD patients responded well to Vedolizumab treatment, with 50\% TNF exposed pts achieving clinical remission, with 20-30\% endoscopic healing rates. Although 20\% pts needed surgery, we have shown efficacy of Vedolizumab in CD to be as good as UC, with a better outcome in anti TNF naïve patients.

\section{PTH-117 PRACTICE PATTERN VARIABILITY IN THE MANAGEMENT OF ACUTE SEVERE COLITIS: A UNITED KINGDOM PROVIDER SURVEY}

${ }^{1}$ Shaji Sebastian*, ${ }^{1}$ Jessica Lisle, ${ }^{3}$ Sreedhar Subramanian, Nicholas Kennedy. ${ }^{1}$ Hull University Teaching Hospitals NHS Trust, Hull, UK; ${ }^{2}$ Royal Liverpool and Broadgreen University Hospitals NHS Trust, Liverpool, UK; ${ }^{3}$ Royal Devon and Exeter NHS Foundation Trust, Exeter, UK

\subsection{6/gutjnl-2019-BSGAbstracts. 176}

Introduction Lack of comparative trial data on dosing regimens of infliximab rescue therapy in acute severe ulcerative colitis (ASUC) has resulted in variable use of rescue regimes in ASUC with potential impact on clinical outcomes. We aimed to (i) study variability in practice in management of ASUC and (ii) evaluate physician perspectives in decision making in rescue therapy.

Methodology An internet-based survey of members of the IBD section of BSG was conducted. The non-vignette section evaluated provider characteristics. The vignette-based section with linked questions detailing progress of an ASUC patient failing intravenous corticosteroids (IVCS) evaluated diagnostic and therapeutic practices. Data was analysed by descriptive statistics and using Fisher's exact test for categorical variables.

Results The response rate of the survey was 31\% (209/682 IBD section members). The provider characteristics as in table 1. In the scenario of IVCS failure, $82.9 \%$ will use Infliximab rescue with only $1.5 \%$ favouring colectomy. Of those who chose infliximab rescue therapy, 78.4\% favoured standard dose $(5 \mathrm{mg} / \mathrm{kg})$ while $37(21.6 \%)$ favoured a higher dose $(10 \mathrm{mg} /$ $\mathrm{kg}$ ) with reasons cited including low albumin and high CRP. IBD specialists chose higher front-loading dose more often compared to other gastroenterologists $(p=0.01)$

Accelerated Induction was favoured by $51.5 \%$ of the respondents while $25 \%$ preferred standard induction (SI) regime and 19\% favoured colectomy. IBD specialists more often favoured AI compared to other gastroenterologists $(p=0.03)$. The main factor for favouring AI was the presence of predictors of low infliximab trough levels $(73.9 \%)$. The reasons cited by those favouring SI $(n=57)$ included lack of 
evidence for AI (18), their usual practice (11), unlicensed regime (7), and safety concerns (4). 100\% of the respondents who favoured colectomy cited safety concerns as their main reason for deciding against continuing medical therapy.

\begin{tabular}{ll} 
Abstract PTH-117 Table 1 & N (\%) \\
\hline & $41(20.1)$ \\
\hline Job Role & $144(70.6)$ \\
IBD specialist & $21(10.3)$ \\
Gastroenterologist with interest in IBD & \\
Gastroenterologist with main interest in other areas & $82(39.6)$ \\
Type of institution & $126(60.9)$ \\
University teaching hospital & \\
District General hospital & \\
ASUC patients (per year) & $15(7.2)$ \\
$<5$ & $83(39.9)$ \\
$5-9$ & $80(38.5)$ \\
$10-19$ & $30(14.4)$ \\
$>20$ & \\
Colectomy as alternative to IVCS & $32(15.4)$ \\
Yes & $176(84.6)$ \\
No & \\
Provision of Lap colectomy in ASUC & $81(38.9)$ \\
Yes & $86(41.3)$ \\
No & $16(7.7)$ \\
Unsure & $25(12)$ \\
Proposionally & \\
\hline $25 \%-49 \%$ & $22(10.6)$ \\
\hline $50-74 \%$ & $33(15.9)$ \\
\hline
\end{tabular}

Conclusions There is significant variation in practice in the use of infliximab rescue therapy in ASUC. There is an urgent need for development of care pathways to standardise practice.

\section{PTH-118 MUCOSAL TISSUE SHORT CHAIN FATTY ACIDS CONTRIBUTE TO PREDICTION OF POUCHITIS IN RESTORATIVE PROCTOCOLECTOMY}

\footnotetext{
1,2Jonathan Segal ${ }^{*},{ }^{2}$ Magali Sarafian, ${ }^{2}$ Alexandros Pechlivanis, ${ }^{2}$ Ivan Jose Serrano Contreras, ${ }^{2}$ Jerusa Brignardello, ${ }^{2}$ Yih-harn Siaw, 1,2 Lucia Braz, ${ }^{1,2}$ Susan Clark, 1,2Elaine Holmes, ${ }^{1,2}$ Ailsa Hart. ${ }^{1}$ St Marks Hospital, Middlesex, UK; ${ }^{2}$ Imperial College London, London, UK
}

\subsection{6/gutjnl-2019-BSGAbstracts.177}

Background Restorative proctocolectomy is a surgical option in patients with ulcerative colitis who become refractory to medical therapy. Short chain fatty acids (SCFA) are organic fatty acids with 1-6 carbons which arise from bacterial metabolism from carbohydrates entering the colon. Various studies have implicated SCFA in both the development of IBD and flares of IBD. Furthermore, it has been shown that SCFA concentrations are significantly lower in faecal samples from patients with pouchitis when compared with healthy controls.

Our study aimed to assess longitudinal changes in SCFA that occur in a pouch to determine if they can predict or are associated with the development of pouchitis. To date no study has analysed short chain fatty acids in mucosal biopsy tissue from these patients.

Methods Patients who underwent restorative proctocolectomy at a single centre underwent pouchoscopy at the time of restoration of continuity and then every 6 months for a year. Biopsies from the pouch were retrieved from the pouch body. Pouchitis was defined using the pouch disease activity index. The development of pouchitis was assessed at months 6 and 12 months.

Biopsies samples were snap frozen at time of biopsy and stored in $-80^{\circ} \mathrm{C}$. Samples were thawed and weighed. Sterile water and Methyl tertiary-butyl ether with internal standard (IS) were added with a ratio of $20 \mathrm{mg}$ of sample:50 $\mu \mathrm{L}$ of $\mathrm{H} 20: 250 \mu \mathrm{L}$ of $\mathrm{MTBE}$ and IS with a further $4 \mu \mathrm{L}$ of hydrochloric acid added to each sample. $30 \mu \mathrm{L}$ of the polar phase was then placed into silanized Eppendorf tubes. $150 \mu \mathrm{L}$ of derivatiser was added to each sample and the cap of the tube applied immediately. These were then incubated for 45 minutes at $60^{\circ} \mathrm{C}$ in an oven. $70 \mu \mathrm{L}$ from the silanised vial was placed into vial inserts and analysed in the gas chromatography mass spectrometry machine. (GC-MS). SCFA were measured using an Agilent 7000C Triple Quadrupole GC/MS-MS System according to a previously published method. Simca was used for multivariate analysis and T-tests were used for univariate analysis.

Results There were 56 biopsy samples. There were 22 patients (17 males); 16 UC and 6 FAP patients with longitudinal follow up. The median age of the cohort was 40 years (range 20-60 years). Of the UC patients four developed pouchitis within one year.

When comparing UC patients at the time of closure of ileostomy, there were there were significant decreases in caproic acid $(4674 \mu \mathrm{M}$ vs $12217 \mu \mathrm{M} \quad \mathrm{p}<0.01)$, valeric acid $(1580 \mu \mathrm{M}$ vs $3695 \mu \mathrm{M} \mathrm{p}=0.01)$, isovolaric acid $(721 \mu \mathrm{M}$ vs $2940 \mu \mathrm{M} p=0.05)$, isobutyric acid $35072 \mu \mathrm{M}$ vs $76074 \mu \mathrm{M}$ $\mathrm{p}=0.03)$ and lactic acid $(1580 \mu \mathrm{M}$ vs $3732 \mu \mathrm{M} \quad \mathrm{p}=0.02)$ between those who developed pouchitis within a year and those who did not develop pouchitis at 1 year.There were no significant differences detected between UC patients and FAP patients at each time point analysis.

Conclusion The study has suggested that a decrease in SCFA found in the mucosal tissue at time of closure of ileostomy may predict onset of pouchitis within a year. This study is the first to demonstrate that SCFA can be analysed from biopsies. Future studies need to determine factors that may contribute to tissue SCFA levels which may help develop a potential therapeutic target to optimise and potentially reduce the incidence of pouchitis.

\section{PTH-119 HOW ACCEPTABLE IS A 'TREAT TO TARGET' (T2T) APPROACH TO IBD PATIENTS IN CLINICAL REMISSION?}

Jenelyn Carbonell, John Kane, Omer Mandour, Alvin Odouri O'Chieng, Matt Pinder, Rebecca McKay, John Hamlin, Christian Selinger*. Leeds Teaching Hospitals NHS Trust, Leeds, UK

\subsection{6/gutjnl-2019-BSGAbstracts.178}

Background Treatment algorithms for IBD are shifting from traditional symptom based pathways to a 'treat to target' T2T approach aiming for clinical remission and absence of mucosal inflammation. We aimed to establish whether patients with IBD in clinical remission agree to this more intense approach. 